\title{
Laminin $\gamma 3$ plays an important role in retinal lamination, photoreceptor organisation and ganglion cell differentiation
}

\author{
Birthe Dorgau', Majed Felemban ${ }^{1}$, Alexander Sharpe ${ }^{1}$, Roman Bauer ${ }^{2}$, Dean Hallam $\mathbb{0}^{1}$, David H. Steel', \\ Susan Lindsay ${ }^{1}$, Carla Mellough $\mathbb{1}^{1,3}$ and Majlinda Lako ${ }^{1}$
}

\begin{abstract}
Laminins are heterotrimeric glycoproteins of the extracellular matrix. Eleven different laminin chains have been identified in vertebrates. They are ubiquitously expressed in the human body, with a distinct tissue distribution. Laminin expression in neural retina and their functional role during human retinogenesis is still unknown. This study investigated the laminin expression in human developing and adult retina, showing laminin $\alpha 1, a 5, \beta 1, \beta 2$ and $\gamma 1$ to be predominantly expressed in Bruch's membrane and the inner limiting membrane. Laminin-332 and laminin $\gamma 3$ expression were mainly observed in the neural retina during retinal histogenesis. These expression patterns were largely conserved in pluripotent stem cell-derived retinal organoids. Blocking of laminin $\gamma 3$ function in retinal organoids resulted in the disruption of laminar organisation and synapse formation, the loss of photoreceptor organisation and retinal ganglion cells. Our data demonstrate a unique temporal and spatial expression for laminins and reveal a novel role for laminin $\gamma 3$ during human retinogenesis.
\end{abstract}

\section{Introduction}

The extracellular matrix (ECM) is a non-cellular structure that is present in all tissues and can be divided into two separate types, the connective tissue matrix and the basement membrane ${ }^{1}$. The ECM provides structural support and promotes cellular functions including differentiation, adhesion, migration, proliferation, axonal growth and morphogenesis in many tissues ${ }^{1-4}$. Laminins (Lam) are a family of heterotrimeric glycoproteins and part of the $\mathrm{ECM}^{5}$. They are a major component of basement membranes and are important for multiple biological processes ${ }^{4,5}$. Laminins are composed of one $\alpha$, one $\beta$

\footnotetext{
Correspondence: Majlinda Lako (majlinda.lako@ncl.ac.uk)

${ }^{1}$ Institute of Genetic Medicine, Newcastle University, Newcastle upon Tyne, United Kingdom

${ }^{2}$ Institute of Neuroscience, Newcastle University, Newcastle upon Tyne, United Kingdom

Full list of author information is available at the end of the article.

Edited by A. Verkhratsky
}

and one $\gamma$ chain, and at present 11 different laminin chains have been identified in vertebrates: five $\alpha$, three $\beta$ and three $\gamma$ chains $^{6}$. These 11 laminin chains can assemble into at least 16 different laminin isoforms, named according to their trimer composition ${ }^{7}$. All isoforms have a tissue-specific distribution ${ }^{6}$. Mutations in different laminin chains are known to cause several congenital diseases in human including eye abnormalities ${ }^{8-13}$.

Laminin functions have often been associated with embryogenesis. For example, laminin-111 has been shown to play an essential role in primitive ectoderm differentiation $^{14-16}$ and knockdowns of any individual chain of laminin-111 result in lethality ${ }^{15,17,18}$. Laminin-111 and other laminin chains play an important role during optic cup morphogenesis and retinal histogenesis ${ }^{19-21}$, photoreceptor structure and synapse formation ${ }^{20,22,23}$, stability of the inner limiting membrane (ILM) ${ }^{24}$ and retinal ganglion cell (RGC) axonal growth ${ }^{25-29}$. 
Only two studies to date have investigated the temporal and spatial distribution pattern of laminin during retinogenesis in rat and human ${ }^{30,31}$, showing that laminin expression varies between developmental stages and species, however, the laminin distribution in developing human neural retina has not been systematically studied.

Human pluripotent stem cells (hPSCs), including human embryonic stem cells (hESCs) and human induced pluripotent stem cells (hiPSCs), offer opportunities for research in human embryogenesis, disease modelling and cell replacement therapies. Several reports have shown the successful generation of selforganising retinal organoids from mouse embryonic stem cells and hPSCs under three-dimensional (3D) culture conditions ${ }^{32-38}$. These retinal organoids recapitulate many aspects of in vivo retinogenesis ${ }^{19,39,40}$ and comprise key retinal cell types, which respond to light and electrophysiological stimuli ${ }^{41-44}$.

In this study, we set out to investigate the temporal and spatial distribution of most laminin chains in different species and, importantly, during human retinal development and hPSC-derived retinal organoids. Our data show a distinct temporal and spatial expression pattern for each laminin during human retinogenesis and reveal an important role for Lamy3 in retinal lamination, organisation of the ONL and differentiation of RGCs in hPSCderived retinal organoids.

\section{Materials and methods}

\section{Tissue preparation for Immunohistochemistry}

In this study mouse, macaque and human eye tissue was prepared for experiments following identical protocols. Adult female and male wild-type mice (C57BL/6) obtained through the Functional Genetic Unit (Institute of Genetic Medicine) at Newcastle University were sacrificed by cervical dislocation and the eyes enucleated. Adult macaque eyes were obtained from Newcastle University following guidelines approved by the ethics committee at Newcastle University and carried out in accordance with the guidelines of the UK Home Office, under control of the Animals (Scientific Procedures) Act 1986. Post mortem adult human eyes donated from one 71- and one 86-year-old male were obtained through Manchester Eye Bank, Manchester Royal Eye Hospital. Human embryonic/foetal eyes aged between 6.3 and 18 post-conception weeks ( $\mathrm{PCW}$ ) were obtained from the MRC/Wellcome Trust funded Human Developmental Biology Resource at Newcastle University (HBDR), with appropriate maternal written consent and approval from the Newcastle and North Tyneside NHS Health Authority Joint Ethics Committee. The HDBR is regulated by the UK Human Tissue Authority (HTA) and operates in accordance with the relevant HTA Codes of Practice.
The cornea and lens were removed from all eyes and the remaining posterior eyecups were fixed in $4 \%$ paraformaldehyde (PFA; Santa Cruz Biotechnologies) for 20 minutes-12 hours depending on the tissue size. After several washing steps in physiological phosphate-buffered saline (PBS; $\mathrm{pH} 7.4$ ), eyecups were cryoprotected in PBS containing $30 \%$ sucrose overnight at $4{ }^{\circ} \mathrm{C}$ and embedded in OCT (Optimal Cutting Temperature) compound(Cell Path Ltd, Newtown, UK). Sagital sections were cut on a cryostat (Leica Cm1860). The thickness of the section was dependent on the tissue: $20 \mu \mathrm{m}$ for mouse and monkey eyes, $30 \mu \mathrm{m}$ for adult human eyes and $10-12 \mu \mathrm{m}$ for human embryonic/foetal eyes.

\section{hPSC culture and retinal organoid differentiation}

Retinal organoids were generated as described previously with some modifications ${ }^{37}$. Briefly, hESCs (H9; WiCell Inc.) and hiPSCs derived from adult fibroblast $\left(\mathrm{SB}-\mathrm{Ad} 33^{45}\right.$ ) were expanded in $\operatorname{mTESR}^{\mathrm{im}} 1$ (StemCell Technologies, 05850) on growth factor-reduced Matrigel (BD Biosciences, San Jose, CA) coated plates at $37^{\circ} \mathrm{C}$ and $5 \% \mathrm{CO}_{2}$. In contrast to the protocol described by Mellough et al. ${ }^{37}$, the medium was supplemented with $10 \mu \mathrm{M}$ Rock inhibitor (Y-27632 dihydrochloride; Chemdea, Ridgewood, USA) for the first 2 days of differentiation and with $10 \%$ foetal calf serum (FCS; Life Technologies, UK), $\mathrm{T}_{3}$ (40 ng/ml; Sigma-Aldrich, UK), Taurine $(0.1 \mathrm{mM}$; Sigma-Aldrich) and retinoic acid (0.5 $\mu \mathrm{M}$; Sigma-Adrich) after day 18 of differentiation. Retinal organoids were collected at days 35, 90, 150 and 200 of differentiation and processed for immunohistochemistry (IHC) by fixation in 4\% PFA for 20-30 minutes followed by several washing steps in PBS. After cryoprotection in PBS containing 30\% sucrose overnight, organoids were embedded in OCT (Cell Path Ltd, Newtown, UK) and sections $(10 \mu \mathrm{m})$ were cut on a cryostat (Leica Cm1860).

\section{Immunohistochemistry}

Sections were rinsed in PBS and incubated for 1 hour at room temperature in blocking solution (10\% normal goat serum, $0.3 \%$ Triton-X-100 in PBS). All antibodies were diluted in antibody diluent solution [ADS; $1 \%$ bovine serum albumin (BSA), 0.3\% Triton-X-100 in PBS]. Primary antibodies (Table S1) were applied overnight at $4{ }^{\circ} \mathrm{C}$. After several washing steps in ADS, section were incubated with secondary goat antibodies conjugated either to Alexa488 (Life Technologies), Cy3 (Jackson Immuno Research Laboratories) or Alex647 (Life Technologies) for 2 hours at room temperature in the dark. Then sections were washed several times in PBS and mounted with VectaShield (Vector Laboratories, Burlingame, CA) containing Hoechst (Life Technologies). For each antibody, control IHC was carried out by omitting the primary antibody. 


\section{Laminin blocking experiments}

Blocking of Lam 33 was performed at days 43 and 150 of retinal organoid differentiation. For blocking of Lam $\gamma$, the medium was supplemented with a rabbit IgG antilaminin $\gamma 3$ antibody $(10 \mu \mathrm{g} / \mathrm{ml}$; Table S1). Medium was changed every 2-3 days and the experiment was stopped after 7 days. Brightfield images of retinal organoids were taken after every media change using a Zeiss AxioVert1 (Zeiss, Germany) with a $5 \times / 0.15$ air objective. After experiments, retinal organoids were fixed and further processed for IHC as described above, or rinsed in PBS and immediately frozen at $-80^{\circ} \mathrm{C}$ for quantitative reverse transcriptase-PCR (qRT-PCR). For both experiments, two individual experimental replicates were carried out for IHC and three for qRT-PCR. Quantification of Caspase-3 was performed with Image $(\mathrm{NIH}$, Bethesda, MD). The quantitative data included in the results section represent the mean ( \pm SEM) of Caspase-3 expression in control and group treated with Lamy3 antibody.

\section{Image acquisition and analysis}

Images were taken using a Zeiss Axio ImagerZ2 equipped with an Apotome.2 and Zen 2012 blue software (Zeiss, Germany). Scanning of image stacks was performed with either a $20 \times / 0.8$ air objective or a $40 \times / 1.3$ oil immersion objective using a $z$-axis increment of either $0.49 \mu \mathrm{m}$ for $20 \times$ air objective or $0.28 \mu \mathrm{m}$ for $40 \times$ oil immersion objective. Two independent replicates were obtained for adult mouse, monkey, human and embryonic eye tissues, as well as for retinal organoids. In all, 10 to 15 retinal organoids were collected per replicate and 3-5 examples were imaged at each time point at which a representative image is shown here. Final images are presented as a maximum projection and adjusted for brightness and contrast in Adobe Photoshop CS6 (Adobe Systems).

\section{qRT-PCR analysis}

In total, 15-20 retinal organoids were homogenised using a Dounce Tissue Grinder (Sigma-Aldrich, UK) and RNA was extracted using the Promega tissue extraction kit (Promega, USA) as per manufacturer's instructions. In all, $1 \mu \mathrm{g}$ of RNA was reverse transcribed using random primers (Promega, USA). qRT-PCR was performed using a Quant Studio 7 Flex system (Applied Biosystems, USA) with SYBR Green (Promega, USA). Each primer (Table S2) was used at a concentration of $1 \mu \mathrm{M}$, and at a ratio of 50:50 for forward and reverse. The reaction parameters were as follows: $95^{\circ} \mathrm{C}$ for 15 minutes to denature the complementary DNA and primers, 40 cycles of $94^{\circ} \mathrm{C}$ for 15 seconds followed by primer specific annealing temperature for 30 seconds, succeeded by a melt curve. The data were analysed using 2- $\Delta \Delta \mathrm{Ct}$ method. Statistical analysis was done using Prism 6 (GraphPad Software, La
Jolla, CA). All results were validated using Student's $t$-test for paired samples.

\section{Results \\ Distribution of laminins during retinal development and in adult retina}

We examined the distribution of all existing laminin chains in different adult species, human developing retina, as well as hPSC-derived retinal organoids using IHC. Laminin $\alpha 2$ has no retinal-associated expression (except with the retinal vasculature), nor is implicated in any eyerelated disease ${ }^{30,31}$, for this reason it was excluded from this study.

\section{Laminin expression in the adult mouse, macaque and human retina}

A polyclonal antibody that recognises all three chains of laminin-332 showed diverse expression pattern in all three species (Fig. 1). Strong immunoreactivity was found in Bruch's membrane (BrM) and ILM of the mouse retina (Fig. 1a), whereas laminin-332 was expressed throughout the macaque and human retina (Figs. 1b, c). These findings are consistent with expression data previously obtained in human retina ${ }^{31}$. In all species, no immunoreactivity was found for Lam $\alpha 1$ (Figure S1), corroborating previous data obtained in adult rat and human retina ${ }^{31}$. Lama4 is expressed in mouse and macaque $\mathrm{BrM}$, as well as in the ILM of the macaque retina (Figure S1A). This laminin chain is, however, absent in the human retina (Figure S1C). Expression of Lam $\alpha 5, \beta 1$ and $\beta 2$ was found in BrM of mouse retina and no immunoreactivity was seen in the mature macaque and human retina (Fig. 1, S1). Lam 1 expression was observed in BrM of mouse and macaque retina (Figure S1A, B) and human ILM (Figure S1C). Like laminin-332, Lam 33 expression varied across all three species (Fig. 1). In the mouse retina, weak immunostaining was found in the inner nuclear layer (INL) and the ganglion cell layer (GCL; Fig. 1a) whereas prominent staining was seen in the macaque interphotoreceptor matrix (IPM; close to the retinal pigment epithelium (RPE)), INL and inner plexiform layer (IPL; Fig. 1b). Higher magnification of Lam $\gamma 3$ immunostaining in the INL (data not shown) suggests its expression in a specific bipolar cell (BC) type stratifying their axon terminals in the innermost ON layer of the IPL close to the GCL, indicating most likely an ON BC type or Rod BC. However, in human retina Lam $\gamma 3$ revealed a punctate pattern across all three nuclear layers (Fig. 1c).

Laminin-332, Lam $\beta 2$, Lam $\alpha 5$ in the mouse retina (arrowheads; Fig. 1a), as well as Lam $\gamma 1$ expression in the human retina (arrowhead; Figure S1C) was also associated with retinal vasculature. All results are summarised in Table S3. 


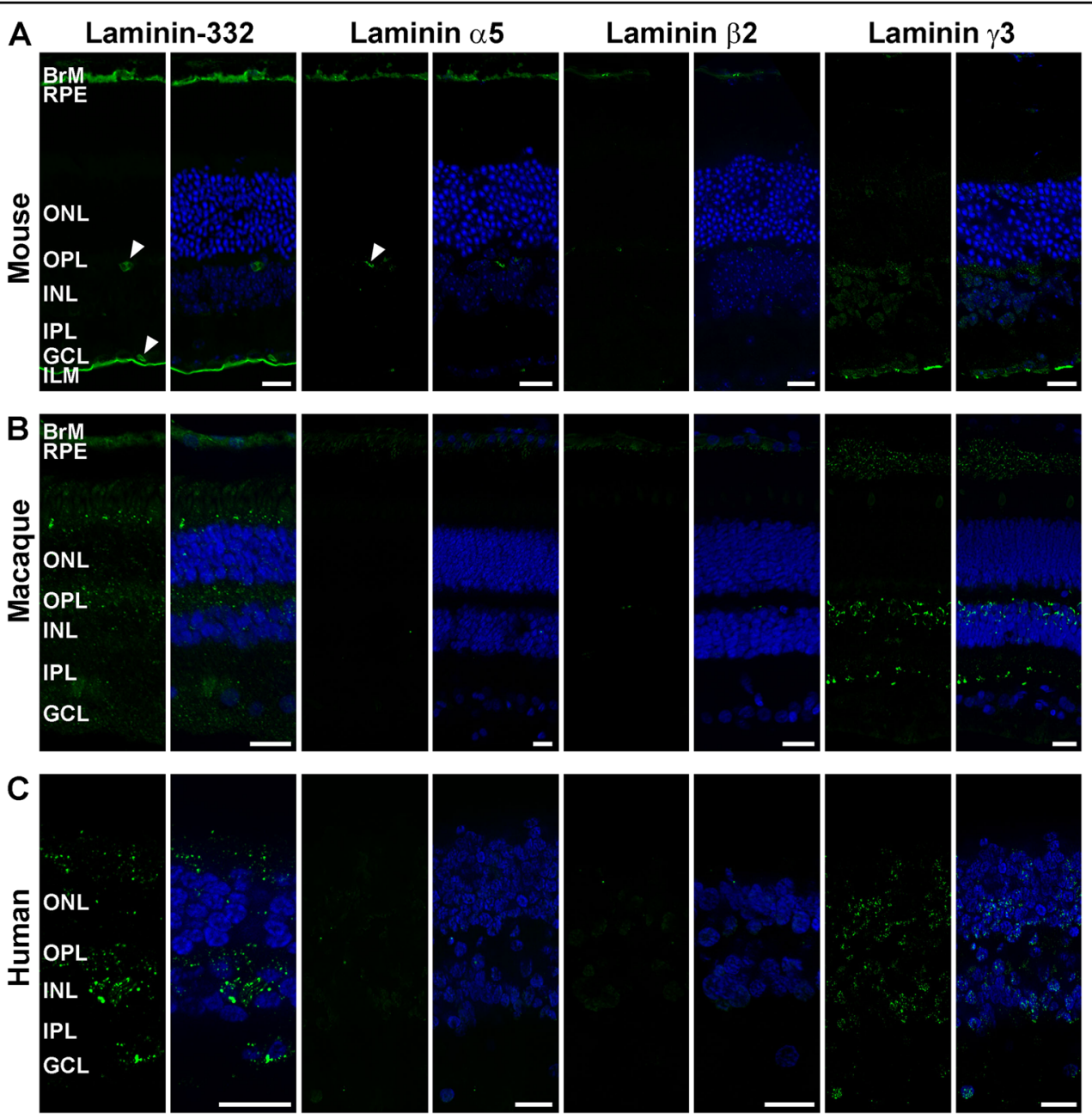

Fig. 1 Expression of laminin-332, laminin $\mathbf{\alpha 5}$, laminin $\mathbf{\beta 2}$ and laminin $\mathbf{3}$ in adult mouse, macaque and human retina. Laminin-332 (green) is expressed in BrM and the ILM of the mouse retina (a), the IPM, OPL and GCL of the macaque retina (b) and in ONL, INL and GCL of human retina (c). Laminin a5 (green) and laminin $\beta 2$ (green) were found in BrM of mouse retina (a) and not in macaque (b) and human retina (c). Laminin $\gamma^{3}$ (green) is expressed in INL and GCL of mouse retina (a), in IPM, INL and IPL of macaque retina (b) and in ONL, INL and GCL of human retina (c). Nuclei are counterstained with Hoechst (blue). Arrowheads indicate expression of laminin-332 and laminin a5 in blood vessels in the mouse retina (a). BrM Bruch's membrane, RPE retinal pigment epithelium, IPM interphotoreceptor matrix, ONL outer nuclear layer, OPL outer plexiform layer, INL inner nuclear layer, IPL inner plexiform layer, GCL ganglion cell layer, ILM inner limiting membrane. Scale bars, 20 um

\section{Distribution of laminins in human embryonic/foetal retina}

Laminin chain expression in the human retina was examined at several developmental stages from 6.3 to 18 PCW. Laminin-332 was found at all developmental stages studied, revealing expression in BrM and a punctate pattern throughout the neural retina, except for the $10^{\text {th }}$ PCW where expression was observed only in the inner neuroblastic zone (INBZ; Fig. 2a). Later in development (12-18 PCW), laminin-332 immunoreactivity was also observed in the outer neuroblastic zone (ONBZ) and the developing IPM (Fig. 2a). Lam $\alpha 1$ was expressed in BrM and the ILM at 6.3 PCW, was absent at $8 \mathrm{PCW}$ and was found again at $10 \mathrm{PCW}$, but only in BrM (Figure S2A). Starting from the $12^{\text {th }}$ PCW onwards, Lam 1 immunoreactivity was restricted to the ILM, except for the $14^{\text {th }}$ PCW (Figure S2A). Immunoreactivity for Lam $\alpha 4$ was first observed during the $12^{\text {th }}$ PCW, showing expression in BrM and IPM (Figure S2B). At 14 PCW, Lamo4 expression was observed in the IPM and ONBZ; however, no expression was detected at 16 and $18 \mathrm{PCW}$ (Figure S2B). Lam $\alpha 5$ and $\beta 2$ were found in BrM and the ILM throughout all developmental stages (Figs. 2b, c), which is consistent with previously reported data in human foetal retina ${ }^{30}$. In addition, a punctate immunostaining pattern was observed across the entire retina at 12 PCW for Lama5 (Fig. 2b) and in the INBZ at 16 PCW for Lam $\beta 2$ (Fig. 2c). Although the Lam $\beta 1$ expression showed some discrepancies, the expression was generally found in 


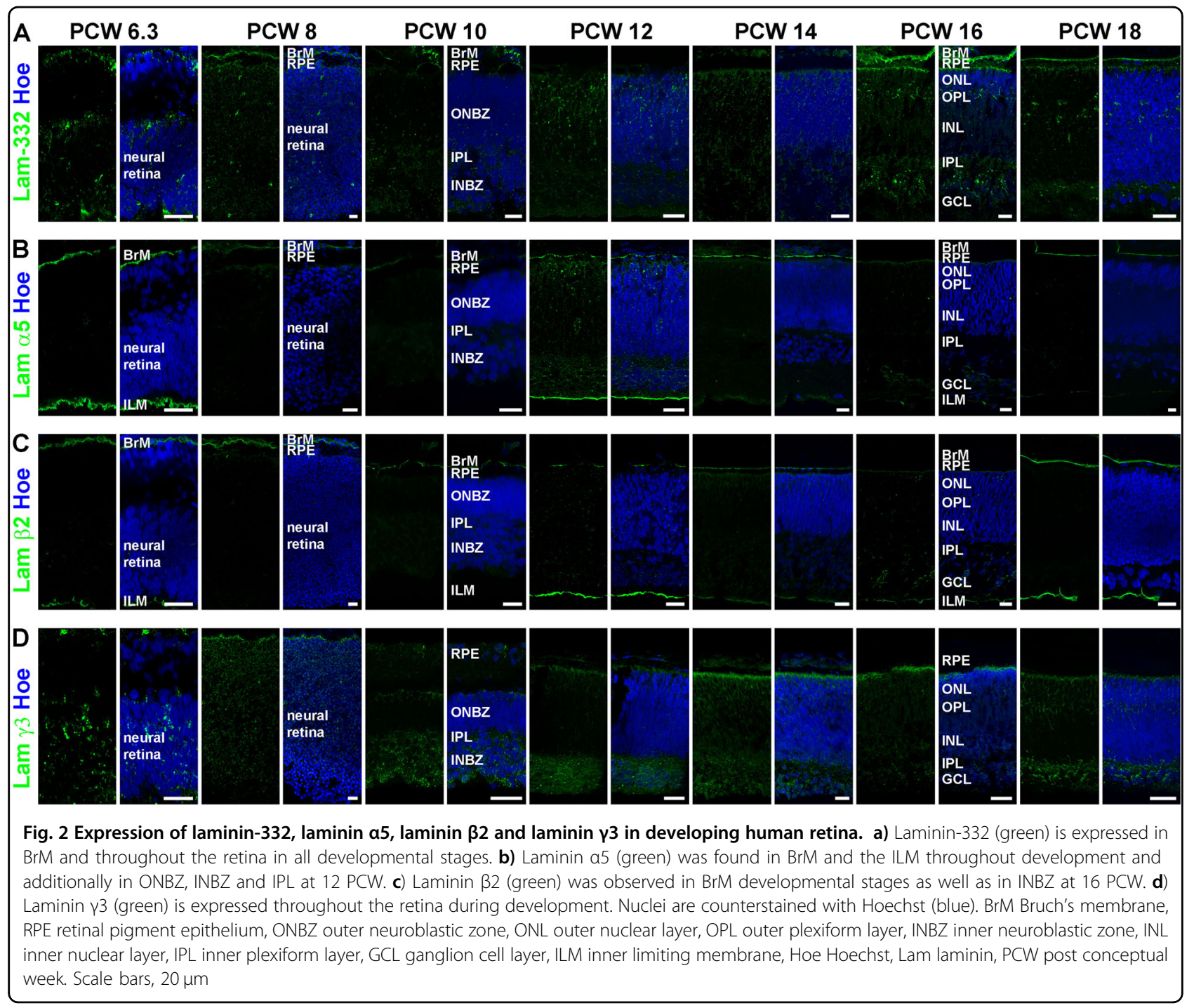

BrM and the ILM from $12 \mathrm{PCW}$ onwards (Figure S2C), corroborating data obtained by Byström et $\mathrm{al}^{30}$.

Immunoreactivity for Lam $\gamma 1$ was found throughout the retina, including the IPM, and the ILM at 6.3 PCW. This expression changed from 8 PCW onwards, revealing Lam $\gamma 1$ in BrM in subsequent developmental stages, except for 10 PCW (Figure S2D). Additionally, Lamy1 was observed in the ILM at 10,12, 16 and 18 PCW (Figure S2D), suggesting maintained expression from the $10^{\text {th }}$ PCW onwards as previously reported ${ }^{30}$. Lam $\gamma 3$ was widely expressed in the retina at all developmental stages studied (Fig. 2d). A punctate pattern throughout the retina was seen at 6.3 and $8 \mathrm{PCW}$. In the 10th and $12^{\text {th }}$ PCW samples, Lamy3 expression was seen in the IPM, IPL and INBZ, whereas Lamy3 immunoreactivity was additionally found in the ONBZ (at 14 and 16 PCW) and later in development (18 PCW) in the outer nuclear layer (ONL; Fig. 2d). All results are summarised in Table S4.
To confirm these findings and investigate the dynamics of laminin chain expression at transcriptional level, we took advantage of a recent RNA-seq study performed by our group ${ }^{46}$ in human developing retina from 4.6 to 18 PCW. This study defined three transcriptional windows: 4.6-7.2 PCW, 7.7-10 PCW and 12-18 PCW, which follow in vivo retinogenesis and correspond to the emergence of optic cup/RPE/lens, RGCs, and photoreceptors/ interneurons/Müller cells (MCs), respectively. Analysis of gene expression showed a significant downregulation of Lam $\alpha 1, \alpha 3, \alpha 4, \beta 1, \beta 2$ and $\gamma 1$ from the first (4.6-7.2 $\mathrm{PCW})$ to the second developmental window (7.7-10 PCW), suggesting an important role for these laminin chains in the very early event associated with optic cup, RPE and lens emergence (Figure S3A-C,E,F,H). Notwithstanding this, a few laminin genes namely Lam $\alpha 1, \alpha 3$ and $\beta 1$ showed a significant upregulation from the second to the third developmental window (12-18 PCW), which 
A
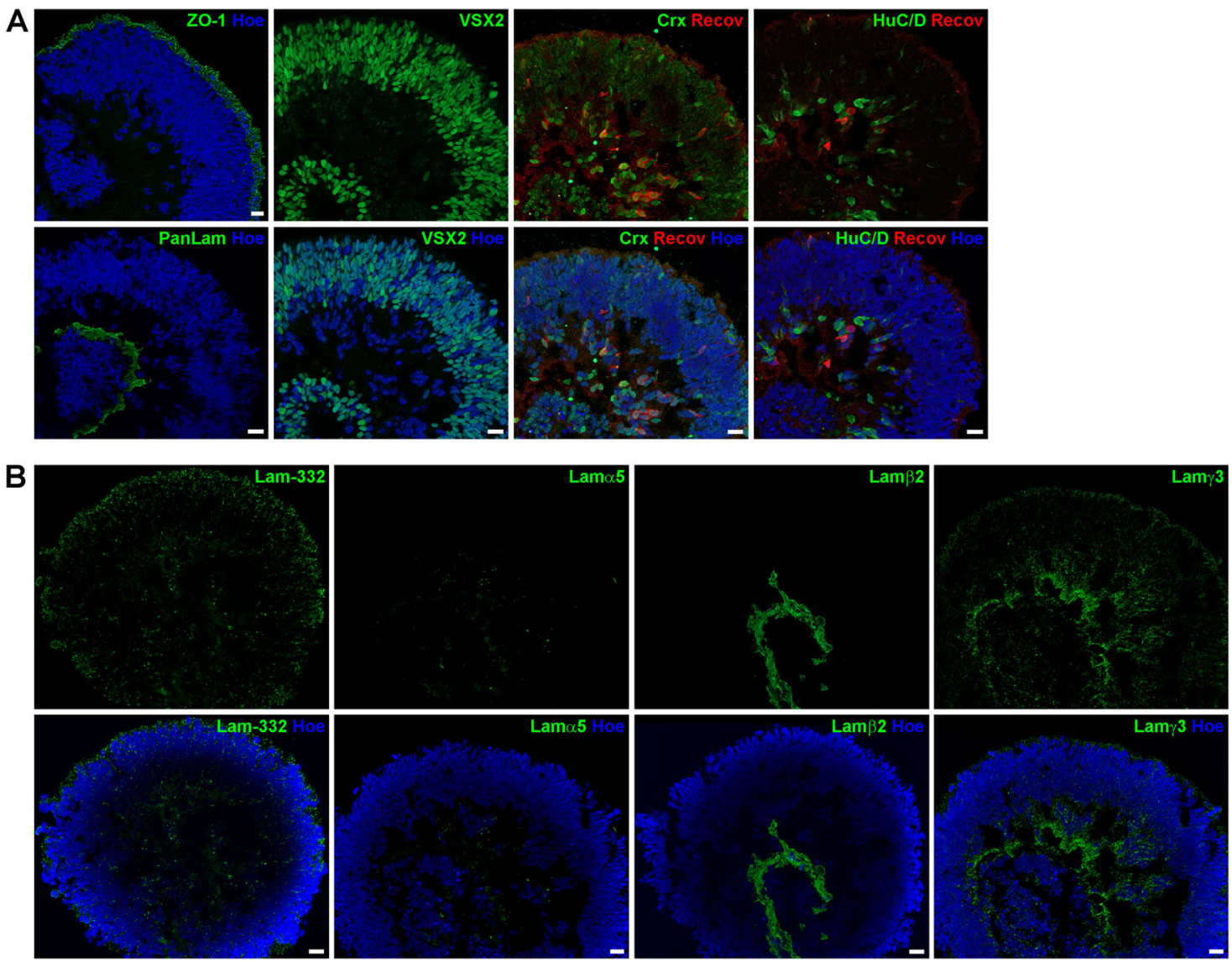

Fig. 3 Expression of retinal markers and laminin-332, laminin a5, laminin $\beta 2$ and laminin $\gamma 3$ in retinal organoids derived from hESCs at day 35 of differentiation. a) Expression of ZO-1 (green), Pan-Laminin (PanLam, green), retinal progenitor cells (VSX2, green), photoreceptors (CRX, green; Recoverin, red) and ganglion cells (HuC/D, green). b) Laminin-332 (green) is expressed throughout the retinal organoid. No immunoreactivity for laminin a5 (green) was found. Laminin $\beta 2$ (green) was observed in a basement membrane-like structure at the basal site of the retinal organoid. Laminin $\gamma 3$ (green) is expressed throughout the retinal organoid with a prominent labelling at the basal site. Nuclei are counterstained with Hoechst (blue). Hoe Hoechst, Lam laminin, PanLam Pan-Laminin, Recov Recoverin. Scale bars, 20 mm

highlights their potential involvement in retinal cell differentiation (Figure S3A,B,E). In contrast, Lamy1-3 were significantly downregulated from the second to the third developmental window (Figure S3H-J), which may be due to their restricted expression pattern in certain areas of the developing retina as shown by IHC (Fig. 2d, S2D).

\section{Expression of laminins in retinal organoids derived from hESC and hiPSC}

Retinal organoids were generated from hESCs/hiPSCs and collected at four different time points during differentiation to assess retinal development and laminin distribution. Retinal organoids derived from hPSCs revealed a clear apical-basal polarity as demonstrated by the apical expression of ZO-1 and basal expression of Pan-Laminin (Fig. 3a). VSX2, a marker for retinal progenitor cells, was mainly expressed in the apical layer of organoids, forming a large layer of retinal progenitor cells at days 35 and 90 of differentiation (Figs. 3a, 4a). In contrast, RGCs and amacrine cells (ACs) detected by the marker protein $\mathrm{HuC} / \mathrm{D}$ were found in the basal region close to the centre of the retinal organoids at day 35 of differentiation (Fig. 3a). Immunoreactivity for CRX, a post-mitotic photoreceptor marker, was observed in cells located both in basal and apical region of retinal organoids (Fig. 3a). Recoverin (a marker for photoreceptors) was found in a few cells in the basal region of the organoids at day 35 (Fig. 3a). The expression pattern of both markers changed dramatically over time, showing an enormous increase in CRX- and Recoverin-positive cells at day 90 (Fig. 4a). At this differentiation stage, two bands of CRX- and Recoverin-positive cells were found in organoids, both in the apical and basal side (Fig. 4a). At days 150 and 200 of differentiation, cells expressing CRX and Recoverin were mainly located in the apical region of retinal organoids, forming a thick putative ONL (Figs. 5a, 6a). HuC/Dpositive cells were located at the basal aspect of retinal organoids at day 150 (Fig. 5a) and 200 (Fig. 6a). At both 

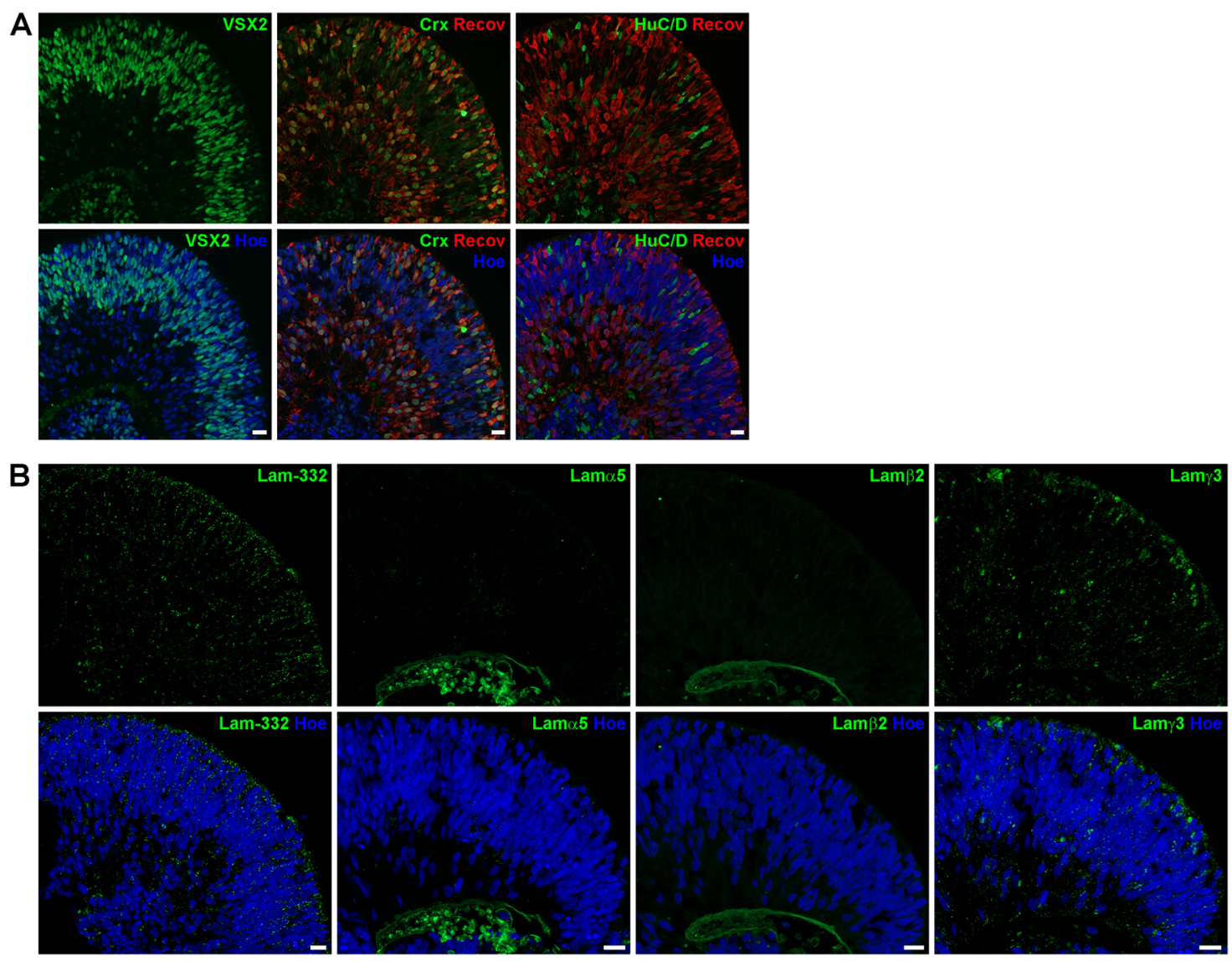

Fig. 4 Expression of retinal markers and laminin-332, laminin $\alpha 5$, laminin $\beta 2$ and laminin $\gamma 3$ in retinal organoids derived from $h E S C s$ at day 90 of differentiation. a) Expression of retinal progenitor cells (VSX2, green), photoreceptors (CRX, green; Recoverin, red) and ganglion cells (HuC/D, green). b) Laminin-332 (green) and laminin $\gamma 3$ are expressed throughout the retinal organoid with a more prominent labelling at the apical site. Laminin a5 (green) and laminin $\beta 2$ (green) was observed in a basement membrane-like structure at the basal site of the retinal organoid. Nuclei are counterstained with Hoechst (blue). Hoe Hoechst, Lam laminin, Recov Recoverin. Scale bars, $20 \mu \mathrm{m}$

time points, retinal organoids contained all retinal cell types including rod and cone photoreceptors (Fig. 6a), BCs, horizontal cells (HCs) and ACs (data not shown), as well as the MCs (Fig. 5a).

Laminin-332 was expressed in all developmental stages in hESC- (Figs. 3b, 6b) and hiPSC-derived retinae (Figure S6), showing a punctate pattern across the entire retinal organoids. This pattern was more intense apically at day 35 of differentiation (Fig. 3b). At day 90, laminin-332 puncta were also seen around photoreceptors as indicated by double staining with CRX (Figure S5A) in the developing ONL (Fig. 4b). Laminin-332 expression was observed throughout the neural retina on day 150 of differentiation (Fig. 5b). Apical laminin-332 immunoreactivity colocalised with RBP3, a marker specific for the IPM, confirming its expression in the IPM of retinal organoids (Figure S5B). Similar to day 150, at day 200, laminin-332 expression was observed through the neural retina, with more prominent expression in the putative developing OPL (Fig. 6b), the future site where synaptic connects between photoreceptors and second-order neurons will form.

Lam 1 was found in a basal membrane-like structure throughout differentiation in retinal organoids derived from hESCs (Figure S4). Unlike hESCs, Lam $\alpha 1$ expression in hiPSC-derived retinal organoids revealed a strong expression in a small number of cells in the developing ONL (Figure S6) and in a basement membrane-like structure, which started to form at days 90 and 120 of differentiation (arrowheads, Figure S6). Throughout all stages, no Lama4 immunoreactivity was seen either in hESC- (Figure S4) or in hiPSC-derived retinae (Figure S6). Lam $\alpha 5$ and $\beta 2$ expression shared a similar expression pattern in a basement membrane-like structure of retinal organoids derived from hESCs (Figure S3 and S6B) and hiPSCs (Figure S6), with clear Lam $\beta 2$ expression observed from day 35 and Lam $\alpha 5$ from day 90 onwards. Lam $\beta 1$ immunoreactivity revealed a punctate pattern apically in the developing ONL at differentiation day 90 in both hESC- and hiPSC-derived retinae (Figure S4B, S6B). Later 

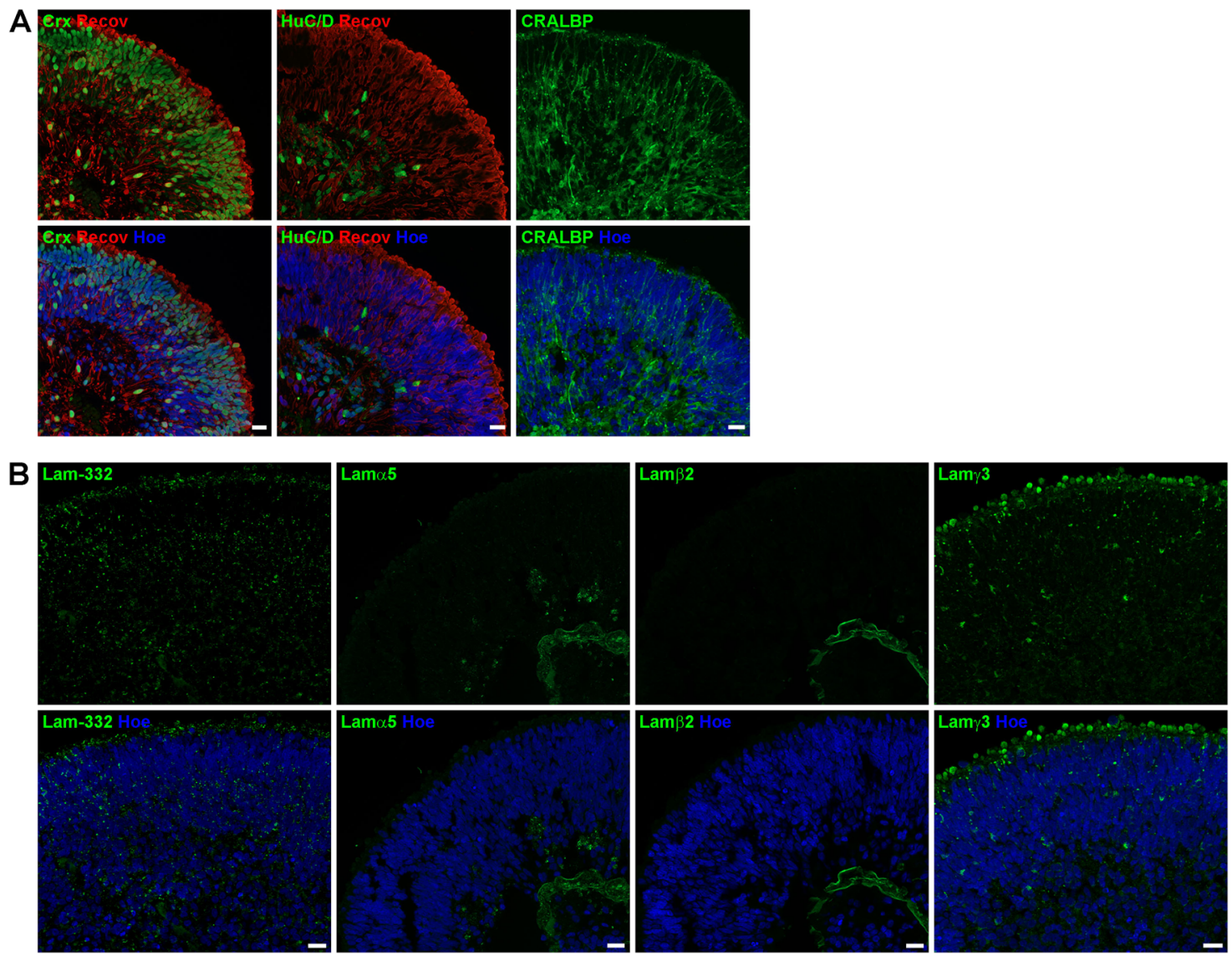

Fig. 5 Expression of retinal markers and laminin-332, laminin $\alpha 5$, laminin $\beta 2$ and laminin $\gamma 3$ in retinal organoids derived from hESCs at day 150 of differentiation. a) Expression of photoreceptors (CRX, green; Recoverin, red), ganglion cells (HuC/D, green) and Müller cells (CRALBP, green). b) Laminin-332 (green) is expressed throughout the retinal organoids. Laminin a5 (green) and laminin $\beta 2$ (green) were observed in a basement membrane-like structure at the basal site of the retinal organoid. Laminin $\gamma 3$ (green) was found at the apical site of retinal organoid with a prominent labelling above cell nuclei, most likely in the IS of photoreceptors. Nuclei are counterstained with Hoechst (blue). Hoe Hoechst, IS inner segments, Lam laminin, Recov Recoverin. Scale bars, $20 \mu \mathrm{m}$

at day 200 for hESC-derived retinal organoids and at day 120 for hiPSC-derived retinae, Lam $\beta 1$ expression was observed in a basal basement membrane-like pattern at the centre of organoids (Figure S4D, S6C). Lam $\beta 2$ and $\gamma 1$ were found in a basal basement membrane-like structure in organoids derived from both hPSC cell lines throughout development (Figs. 3, 6b, S4,S6). Double staining with Lam $\beta 2$ and Collagen IV, a component of basement membranes, revealed colocalisation, confirming that Lam $\beta 2$ is expressed in a basement membrane of organoids (Figure S5A).

At all developmental stages, Lamy3 showed a punctate pattern across the entire retinal organoid derived from hESCs (Figs. 3, 6b) and hiPSCs (Figure S6). At day 35 of differentiation, this pattern was more prominent in the basal aspect of the neural retina (Fig. 3b), whereas at day 90 Lam $\gamma 3$ immunoreactivity showed weak basal expression (Fig. 4b) and instead revealed strong expression at the apical surface where developing photoreceptors are located (Figure S5A). Lamy3 expression in hiPSC-derived retinal organoids showed a comparable distribution pattern to retinal organoids derived from hESCs (Figure S6). At day 150, Lamץ3 was found in the layer apical to the developing ONL in hESCs-derived retinal organoids (Fig. 5b), indicating expression in the developing inner segments (ISs) of photoreceptors. In addition, doublelabelling with antibodies against Lam $\gamma 3$ and RBP3 confirmed that Lam $\gamma 3$ is also expressed in the IPM (Figure S5B). At day 200, the Lamy3 immunoreactivity revealed the same pattern as in day 150, although it was stronger than at earlier differentiation time points (Fig. 6b).

\section{Effects of neutralising Laminin $\gamma 3$ in human retinal development}

To investigate the role of Lamy3 during hESC differentiation, an antibody was used to neutralise Lamy3 function at two different developmental time points. Blocking of Lamy3 at day 43 induced degeneration of the 

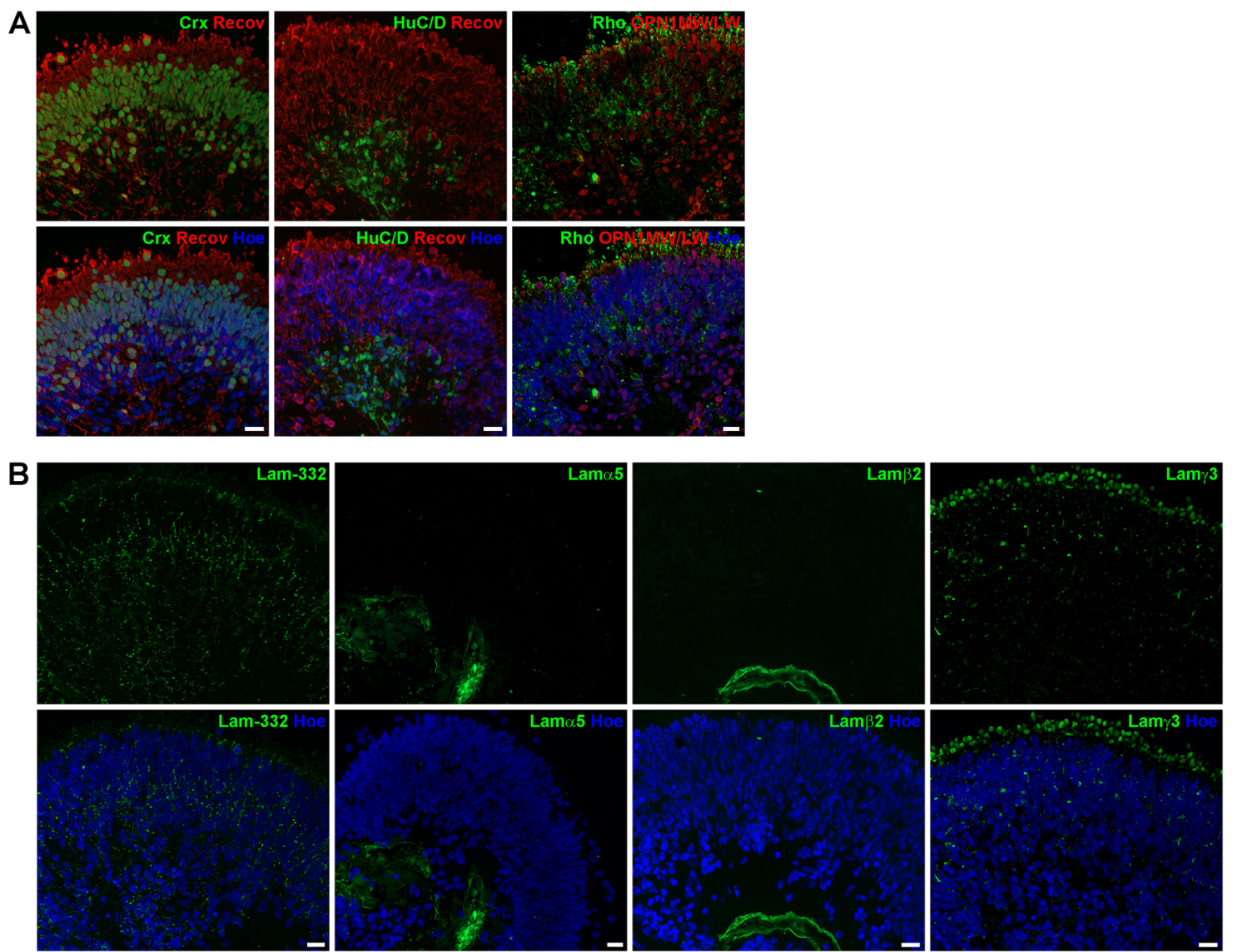

Fig. 6 Expression of retinal markers and laminin-332, laminin a5, laminin $\beta 2$ and laminin $\gamma 3$ in retinal organoids derived from hESCs at day 200 of differentiation. a) Expression of photoreceptors (CRX, green; Recoverin, red), ganglion cells (HuC/D, green), rods (Rho, Rhodopsin, green) and cones (OPN1MW/OPN1LW, red). b) Laminin-332 (green) is expressed throughout the retinal organoid with a prominent labelling in the middle of the organoid. Laminin a5 (green) and laminin $\beta 2$ (green) were observed in a basement membrane-like structure at the basal site of the retinal organoid. Laminin $\gamma 3$ (green) was found predominantly at the apical site of retinal organoid with a prominent labelling above cell nuclei, most likely in the IS of photoreceptors. Nuclei are counterstained with Hoechst (blue). Hoe Hoechst, IS inner segments, Lam laminin, Recov Recoverin, Rho Rhodopsin. Scale bars, $20 \mu \mathrm{m}$

bright phase retinal neuroepithelial structures observed at the periphery of organoids over time compared with control retinal organoids (Fig. 7a). The control organoids showed the expected expression of Recoverin, CRX and VSX2, including a thick apical layer of VSX2-positive cells and almost all Recoverin- and CRX-positive cells in the inner retina (Fig. 7b, control). Although blocking Lamy3 still resulted in detection of the aforementioned markers, the retina-like structural organisation was disrupted, showing disorganised and reduced expression of VSX2, Recoverin and CRX, as well as $\mathrm{HuC} / \mathrm{D}$ (Fig. 7b) and SMI-32 (Figure S7B). These findings were confirmed by qRT-PCR results, which revealed a decrease in VSX2 expression and a significant reduced expression of $C R X$ and MATH5 (an RGC marker) under blocking conditions (Figure S7A). Expression of caspase-3, an apoptosis marker, was increased under blocking condition (31.34 \pm
$3.51 \%)$ compared with control group (8.11 $\pm 1.38 \%$; Figure S7B).

Similar findings were obtained by blocking Lamy3 later at day 150. Under blocking conditions, the retinal neuroepithelium at the periphery of organoids degenerated over time while that of control organoids remained intact (Fig. 7c). Ganglion cell bodies (detected by $\mathrm{HuC} / \mathrm{D}$ immunostaining) and ganglion cell dendrites (detected by SMI-32 immunostaining) were observed in the inner retina in control organoids (Figs. 7d, e). No ganglion cell bodies were detected (Fig. 7d) under the blocking conditions, but a few putative remaining dendrites were still found (arrowheads, Fig. 7e). In accordance, qRT-PCR data revealed a significant reduction in the expression of MATH5 (Figure S7C). Caspase-3 expression was also increased under blocking conditions $(20.99 \pm 5.4 \%)$ compared with control group $(1.1 \% \pm 0.16)$. The Caspase- 3 positive cells were found throughout organoids, including the basal layer 


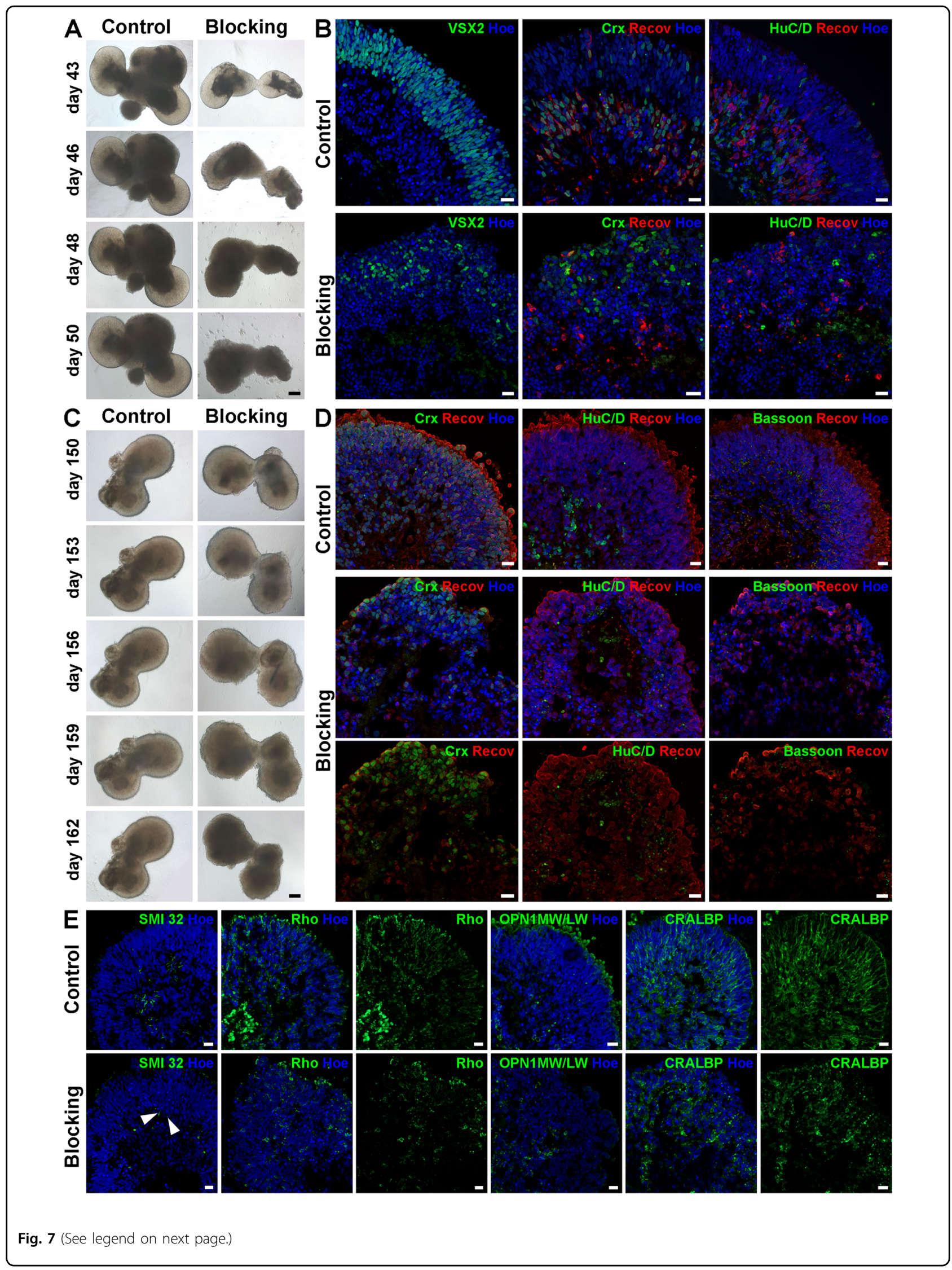


(see figure on previous page)

Fig. $\mathbf{7}$ Blocking of laminin $\mathbf{3}$ in retinal organoids derived from hESCs at day $\mathbf{4 3}$ and day $\mathbf{1 5 0}$ of differentiation. a) Brightfield images revealed a degeneration of bright phase retinal neuroepithelial structures at the apical edge of the organoids in the blocking condition over time in the early blocking experiment (day 43). b) Expression of retinal progenitor cells (VSX2, green), photoreceptors (CRX, green; Recoverin, red) and ganglion cells (HuC/D, green) showed a reduction in cell number and a disruption of the lamination in retinal organoids after blocking of laminin $\gamma 3$ at day 43 of differentiation. c) Brightfield images indicated a degeneration of the retinal neuroepithelial structures at the edge of organoids over time upon blocking of laminin $\gamma_{3}$ at day 150 of differentiation. d) Expression of photoreceptors (CRX, green; Recoverin, red) indicated the lamination loss in retinal organoids after blocking of laminin $\gamma 3$ at day 150 of differentiation. Expression of ganglion cells (HuC/D; green) were absent in the blocking condition. Bassoon (green) expression is significantly decreased in blocked-retinal organoids. e) After laminin $\gamma 3$ blocking only a few ganglion cell dendrites detected by SMI-32 (green) were still present (arrowheads). Expression of rods (Rho, green), cones (OPN1MW/OPN1LW, green) and Müller cells (CRALBP, green) revealed the disrupted lamination of retinal organoids and a reduction in their cell number at day 150 of differentiation. Nuclei are counterstained with Hoechst (blue). Hoe Hoechst, Recov Recoverin, Rho Rhodopsin. Scale bars, 50 um for a and c; $20 \mu m$ for b, d, e

where ganglion cells are located (Figure S7D). Together these findings indicate that that ganglion cells may undergo apoptosis in response to Lamy3 neutralisation. The number of photoreceptor precursors decreased in Lam 33 blocked cultures as demonstrated by an immunolabelling with Recoverin and CRX (Fig. 7d). Moreover, in the blocking condition photoreceptors were disorganised compared with those in control organoids where developing photoreceptors formed a thick putative ONL (Fig. 7d). This was also reflected in the expression pattern of Rhodopsin, which was reduced and disorganised in organoids incubated with blocking antibody to Lamy3 (Fig. 7e). Opsin immunoreactivity was weak and only found in the basal part of the retinal organoids for OPN1MW/ OPN1LW (Fig. 7e) and almost absent for OPN1SW under blocking conditions (Figure S7D). In contrast, control organoids revealed a prominent OPN1MW/OPN1LW immunolabelling in putative photoreceptor IS adjacent to the ONL (Fig. 7e) and OPN1SW expression in cells located in the basal part of retinal organoids (Figure S7D). These findings were confirmed at gene expression level, showing a significant decrease in the expression of Recoverin, Rhodopsin and Opsins (Figure S7C). In control, retinal organoids punctate Bassoon labelling was found in the middle and in the basal part of organoids colocalised with Recoverin, suggesting that structural elements of the ribbon synapses are present (Fig. 7d). This pattern was significantly reduced under blocking conditions (Fig. 7d). In addition, Syntaxin immunoreactivity was also reduced under blocking conditions (arrowhead, Figure S7D), which was also corroborated by qRT-PCR data (Figure S7C). CRALBP (MCs/RPE), AP2 $\alpha$ (ACs) and Prox1 (HCs) were used to assess any effects of blocking Lam 3 on other retinal cell types. In control retinal organoids, CRALBP immunoreactivity spanned the retinal neuroepithelium in a radial pattern (Fig. 7e), whereas this pattern was disrupted in retinal organoids under blocking conditions (Fig. 7e). These changes were also reflected at gene expression level (Figure S7C). The number of AP2 $\alpha$-positive cells was reduced in retinal organoids in the blocking condition compared with control organoids (Figure S7D). In accordance, a significant downregulation of $A P 2 \alpha$ was observed by qRT-PCR (Figure S7C). No changes between control and blocking condition were found for $\mathrm{HCs}$ at protein (Figure S7D) and gene expression level (data not shown). Interestingly, RBP3, a marker for the IPM, showed a significant reduction at both protein and gene expression level (Figure S7C, D).

\section{Discussion}

This study provides a detailed and comprehensive expression analysis for the majority of existing laminin chains in adult mouse, macaque and human retina, developing human eye and retinal organoids derived from hPSCs. All laminin chains displayed unique and distinct temporal and spatial distribution in the adult retina and developing human eye, revealing expression of Lam $\alpha 1, \alpha 5$, $\beta 1, \beta 2$ and $\gamma 1$ predominantly in retinal basement membranes and expression of laminin-332 and Lamy3 throughout the retina. hPSC-derived retinal organoids mimicked laminin expression during early human retinal development. Furthermore, the study demonstrates for the first time that Lam $\gamma 3$ is essential for retinal lamination, photoreceptor organisation, differentiation of RGCs, as well as synapse stability during human retinogenesis.

Lam $\gamma 3$, is broadly expressed in skin, heart, lung, reproductive tracts, brain and retina, ${ }^{9,10}$. Although a single ablation of either Lam $\gamma 3$ or $\operatorname{Lam} \beta 2$ causes minimal or almost no changes in retinal morphology in other stu$\operatorname{dies}^{24,48,49}$, double-knockout of both laminin chains results in defects of the ILM formation during retinal development, leading to retinal dysplasia ${ }^{24}$. Furthermore, a deletion of both Lam $\beta 2$ and $\gamma 3$ was shown to impair MC maturation and physiological function ${ }^{50}$. We used the retinal organoid model to block the function of Lam 33 both early (day 43) and late (day 150) time points in development when retinal lamination is more advanced and multiple retinal cell types are present. Blocking of Lam 33 function early in development resulted in disruption of laminar organisation and a significant reduction in the expression of retinal progenitor marker (VSX2), photoreceptor marker (CRX and Recoverin) and ganglion/ACs ( $\mathrm{HuC} / \mathrm{D})$. Later in development similar results were obtained, showing disrupted laminar organisation and reduced expression of 
specific marker proteins for photoreceptors, ACs and the synaptic markers. Amacrine and ganglion cell marker protein $\mathrm{HuC} / \mathrm{D}$ was absent and only a few dendritic structures were observed in blocked-retinal organoids, suggesting an essential role for this laminin chain in ganglion cell differentiation. The ribbon synapse marker protein, Bassoon, and Syntaxin, a presynaptic marker protein, were significantly reduced upon Lam 3 blocking, suggesting that Lamy 3 might be involved in synapse stability/formation at this stage of development. The disorganisation of MCs, which form the ILM by their end-feet processes, could be a reason for the reduction in RGCs. Supporting evidence come from Halfter et al. ${ }^{51}$, showing that defects in, or absence of an ILM, leads to apoptosis of RGCs.

Ribbon synapse formation during retinal development was also affected after blocking of Lamy3. To date only Lam $\beta 2$ has been associated with the formation of synapses either in the peripheral nervous system ${ }^{52,53}$ or central nervous system, including the retina ${ }^{31,54,55}$. Mouse lacking Lam $\beta 2$ showed dysmorphic photoreceptor synapses ${ }^{56}$. Recently, it was shown that interaction between Lam $\beta 2$ and integrins such as integrin $\alpha 3 \beta 1$, is important for synaptic ribbon anchoring at the photoreceptor synapse and that the dystrophin/dystroglycan complex might play a key role in synaptic formation ${ }^{55}$. Integrins act as receptors for many laminins ${ }^{57}$. As Lamy3 lacks the binding domain for integrins $^{58}$ it is impossible to act via integrins; however, Lamy3 may interact via non-integrin binding proteins such as entactins and dystroglycans, which have been shown to be important for the photoreceptor synapses ${ }^{59}$. Our data suggest that in addition to Lam $\beta 2$, Lam $\gamma 3$ plays a key role in the formation and/or stabilisation of the photoreceptor synapses, acting perhaps via a non-integrin binding protein such as dystroglycan.

In summary, our data provide a comprehensive laminin expression analysis in mouse, macaque and developing and adult human retina and indicate a novel function for Lamy3 in retinal lamination, organisation of the ONL and differentiation of RGCs in hPSC-derived retinal organoids.

\section{Acknowledgements}

This work was funded by the ERC Consolidator award (grant number \#614620), the RPFB Innovation award (grant number \#GR584), NCR Crack-iT retinal challenge (NC/CO16206/1), MRC Confidence in Concept award (MC_PC_15030) and the MRC-Wellcome Trust Human Developmental Biology Resource for provision of human developmental tissues (grant number \# 099175/Z/12/Z).

\section{Author details \\ ${ }^{1}$ Institute of Genetic Medicine, Newcastle University, Newcastle upon Tyne, United Kingdom. ${ }^{2}$ Institute of Neuroscience, Newcastle University, Newcastle upon Tyne, United Kingdom. ${ }^{3}$ Lions Eye Institute, Centre for Ophthalmology and Visual Science, University of Western Australia, Nedlands WA, Australia}

\section{Authors' contributions}

B.D.: manuscript preparation, experimental design and execution, data analysis; M.F.: experimental design and execution; A.S.: performed experiments, data analysis; R.B.: data analysis; D.H.: performed experiments, data analysis; D.H.S., S. L., C.M.: sample collection, experimental design and fund raising; M.L.: experimental design, data analysis, fund raising and manuscript preparation. All authors approved the final version of the manuscript.

Conflict of interest

The authors declare that they have no conflict of interest.

\section{Publisher's note}

Springer Nature remains neutral with regard to jurisdictional claims in published maps and institutional affiliations.

Supplementary Information accompanies this paper at (https://doi.org/ 10.1038/s41419-018-0648-0).

Received: 6 March 2018 Revised: 12 April 2018 Accepted: 26 April 2018 Published online: 23 May 2018

\section{References}

1. Bonnans, C., Chou, J. \& Werb, Z. Remodelling the extracellular matrix in development and disease. Nat. Rev. Mol. Cell Biol. 15, 786-801 (2014).

2. Frantz, C., Stewart, K. M. \& Weaver, V. M. The extracellular matrix at a glance. J. Cell Sci. 123, 4195-4200 (2010)

3. Hynes, R. O. Stretching the boundaries of extracellular matrix research. Nat. Rev. Mol. Cell Biol. 15, 761-763 (2014)

4. Pozzi, A., Yurchenco, P. D. \& Lozzo, R. V. The nature and biology of basement membranes. Matrix Biol. 57-58, 1-11 (2017).

5. Domogatskaya, A., Rodin, S. \& Tryggvason, K. Functional diversity of laminins. Annu. Rev. Cell Dev. Biol. 28, 523-553 (2012).

6. Durbeej, M. Laminins. Cell Tissue Res. 339, 259-268 (2010).

7. Aumailley, M. et al. A simplified laminin nomenclature. Matrix Biol. 24, 326-332 (2005).

8. Aisenbrey, S. et al. Retinal pigment epithelial cells synthesize laminins, including laminin 5, and adhere to them through alpha3- and alpha6containing integrins. Invest. Ophthalmol. Vis. Sci. 47, 5537-5544 (2006).

9. Koch, M. et al. Characterization and expression of the laminin gamma3 chain: a novel, non-basement membrane-associated, laminin chain. J. Cell Biol. 145, 605-618 (1999).

10. Li, Y. N. et al. The gamma3 chain of laminin is widely but differentially expressed in murine basement membranes: expression and functional studies. Matrix Biol. 31, 120-134 (2012).

11. Matejas, $V$. et al. Mutations in the human laminin beta 2 (LAMB2) gene and the associated phenotypic spectrum. Hum. Mutat. 31, 992-1002 (2010).

12. Sampaolo, S. et al. Identification of the first dominant mutation of LAMA5 gene causing a complex multisystem syndrome due to dysfunction of the extracellular matrix. J. Med. Genet. 54, 710-720 (2017).

13. Wewer, U. M. et al. Laminin beta-2 chain and adhalin deficiency in the skeletalmuscle of Walker-Warburg syndrome (cerebro-ocular dysplasia-muscular dystrophy). Neurology 45, 2099-2101 (1995).

14. Li, X. et al. Fibroblast growth factor signaling and basement membrane assembly are connected during epithelial morphogenesis of the embryoid body. J. Cell Biol. 153, 811-822 (2001).

15. Miner, J. H., Li, C., Mudd, J. L., Go, G. \& Sutherland, A. E. Compositional and structural requirements for laminin and basement membranes during mouse embryo implantation and gastrulation. Development 131, 2247-2256 (2004).

16. Murray, P. \& Edgar, D. Regulation of programmed cell death by basement membranes in embryonic development. J. Cell Biol. 150, 1215-1221 (2000).

17. Smyth, N. et al. The targeted deletion of the LAMC1 gene. Ann. N Y Acad. Sci. 857, 283-286 (1998)

18. Smyth, N. et al. Absence of basement membranes after targeting the LAMC1 gene results in embryonic lethality due to failure of endoderm differentiation. J. Cell Biol. 144, 151-160 (1999).

19. Bryan, C. D., Chien, C. B. \& Kwan, K. M. Loss of laminin alpha 1 results in multiple structural defects and divergent effects on adhesion during vertebrate optic cup morphogenesis. Dev. Biol. 416, 324-337 (2016). 
20. Dong, L. J. \& Chung, A. E. The expression of the genes for entactin, laminin A, laminin B1 and laminin B2 in murine lens morphogenesis and eye development. Differ. Res. Biol. Divers. 48, 157-172 (1991).

21. Kwan, K. M. Coming into focus: the role of extracellular matrix in vertebrate optic cup morphogenesis. Dev. Dyn. 243, 1242-1248 (2014).

22. Hunter, D. D., Llinas, R., Ard, M., Merlie, J. P. \& Sanes, J. R. Expression of S-laminin and laminin in the developing rat central-nervous-system. J. Comp. Neurol. 323, 238-251 (1992).

23. Libby, R. T., Hunter, D. D. \& Brunken, W. J. Developmental expression of laminin beta 2 in rat retina - Further support for a role in rod morphogenesis. Invest. Ophthalmol. Vis. Sci. 37, 1651-1661 (1996).

24. Pinzon-Duarte, G., Daly, G., Li, Y. N., Koch, M. \& Brunken, W. J. Defective formation of the inner limiting membrane in laminin beta 2-and gamma 3null mice produces retinal dysplasia. Invest. Ophthalmol. Vis. Sci. 51, 1773-1782 (2010).

25. Cohen, J., Burne, J. F., Mckinlay, C. \& Winter, J. The role of laminin and the laminin fibronectin receptor complex in the outgrowth of retinal ganglion-cell axons. Dev. Biol. 122, 407-418 (1987).

26. Morissette, N. \& Carbonetto, S. Laminin alpha 2 chain (M chain) is found within the pathway of avian and murine retinal projections. J. Neurosci. 15, 8067-8082 (1995).

27. Randlett, O., Poggi, L., Zolessi, F. R. \& Harris, W. A. The oriented emergence of axons from retinal ganglion cells is directed by laminin contact in vivo. Neuron 70, 266-280 (2011).

28. Sarthy, P. V. \& Fu, M. Localization of laminin-B1 messenger-RNA in retinal ganglion-cells by in situ hybridization. J. Cell Biol. 110, 2099-2108 (1990).

29. Vecino, E., Heller, J. P., Veiga-Crespo, P., Martin, K. R. \& Fawcett, J. W. Influence of extracellular matrix components on the expression of integrins and regeneration of adult retinal ganglion cells. PLOS ONE 10, e0125250 (2015).

30. Bystrom, B., Virtanen, I., Rousselle, P., Gullberg, D. \& Pedrosa-Domellof, F. Distribution of laminins in the developing human eye. Invest. Ophthalmol. Vis. Sci. 47, 777-785 (2006).

31. Libby, R. T. et al. Laminin expression in adult and developing retinae: evidence of two novel CNS laminins. J. Neurosci. 20, 6517-6528 (2000).

32. Boucherie, C. et al. Brief report: self-organizing neuroepithelium from human pluripotent stem cells facilitates derivation of photoreceptors. Stem Cells $\mathbf{3 1}$, 408-414 (2013).

33. Eiraku, M. et al. Self-organizing optic-cup morphogenesis in three-dimensional culture. Nature 472, 51-U73 (2011)

34. Gonzalez-Cordero, A. et al. Photoreceptor precursors derived from threedimensional embryonic stem cell cultures integrate and mature within adult degenerate retina. Nat. Biotechnol. 31, 741 (2013).

35. Kuwahara, A. et al. Generation of a ciliary margin-like stem cell niche from selforganizing human retinal tissue. Nat. Commun. 6, 6286 (2015).

36. Lamba, D. A. et al. Generation, purification and transplantation of photoreceptors derived from human induced pluripotent stem cells. PLOS ONE $\mathbf{5}$ e8763 (2010).

37. Mellough, C. B. et al. IGF-1 signaling plays an important role in the formation of three-dimensional laminated neural retina and other ocular structures from human embryonic stem cells. Stem Cells 33, 2416-2430 (2015).

38. Nakano, T. et al. Self-formation of optic cups and storable stratified neural retina from human ESCs. Cell Stem Cell 10, 771-785 (2012).

39. Gonzalez-Cordero, A. et al. Recapitulation of human retinal development from human pluripotent stem cells generates transplantable populations of cone photoreceptors. Stem Cell Rep. 9, 820-837 (2017).
40. Volkner, M. et al. Retinal organoids from pluripotent stem cells efficiently recapitulate retinogenesis. Stem Cell Rep. 6, 525-538 (2016).

41. Hallam, D. et al. An induced pluripotent stem cell patient specific model of complement factor $\mathrm{H}(\mathrm{Y} 402 \mathrm{H})$ polymorphism displays characteristic features of age-related macular degeneration and indicates a beneficial role for UV light exposure. Stem Cells 35, 2305-2320 (2017).

42. Quadrato, G. et al. Cell diversity and network dynamics in photosensitive human brain organoids. Nature 545, 48-53 (2017).

43. Zhong, X. et al. Generation of three-dimensional retinal tissue with functional photoreceptors from human iPSCs. Nat. Commun. 5, 4047 (2014).

44. Wahlin, K. J. et al. Photoreceptor outer segment-like structures in long-term 3D retinas from human pluripotent stem cells. Sci. Rep. 7, 766 (2017).

45. Melguizo-Sanchis, D. et al. iPSC modeling of severe aplastic anemia reveals impaired differentiation and telomere shortening in blood progenitors. Cell Death Dis. 9, 128 (2018)

46. Mellough, C., et al. An integrated transcriptional analysis of the developing human retina. PLoS Biol. (under review).

47. Halfter, W., Dong, S., Yip, Y. P., Willem, M. \& Mayer, U. A critical function of the pial basement membrane in cortical histogenesis. J. Neurosci. 22, 6029-6040 (2002).

48. Denes, $\mathrm{V}$. et al. Laminin deficits induce alterations in the development of dopaminergic neurons in the mouse retina. Vis. Neurosci. 24, 549-562 (2007).

49. Gnanaguru, G. et al. Laminins containing the beta2 and gamma3 chains regulate astrocyte migration and angiogenesis in the retina. Development $\mathbf{1 4 0}$, 2050-2060 (2013)

50. Hirrlinger, P. G. et al. Genetic deletion of laminin isoforms beta2 and gamma3 induces a reduction in Kir4.1 and aquaporin-4 expression and function in the retina. PLOS ONE 6, e16106 (2011).

51. Halfter, W., Willem, M. \& Mayer, U. Basement membrane-dependent survival of retinal ganglion cells. Invest. Ophthalmol. Vis. Sci. 46, 1000-1009 (2005).

52. Hunter, D. D., Shah, V., Merlie, J. P. \& Sanes, J. R. A laminin-like adhesive protein concentrated in the synaptic cleft of the neuromuscular junction. Nature $\mathbf{3 3 8}$ 229-234 (1989).

53. Noakes, P. G., Gautam, M., Mudd, J., Sanes, J. R. \& Merlie, J. P. Aberrant differentiation of neuromuscular junctions in mice lacking s-laminin/laminin beta 2. Nature 374, 258-262 (1995).

54. Egles, $C$. et al. Laminins containing the beta2 chain modulate the precise organization of CNS synapses. Mol. Cell. Neurosci. 34, 288-298 (2007).

55. Hunter, D. D. et al. CNS synapses are stabilized trans-synaptically by laminins and laminin-interacting proteins. J. Comp. Neurol. https://doi.org/10.1002/ cne.24338 (2017).

56. Libby, R. T., Lavallee, C. R., Balkema, G. W., Brunken, W. J. \& Hunter, D. D. Disruption of laminin beta 2 chain production causes alterations in morphology and function in the CNS. J. Neurosci. 19, 9399-9411 (1999).

57. Belkin, A. M. \& Stepp, M. A. Integrins as receptors for laminins. Microsc. Res. Tech. 51, 280-301 (2000).

58. Ido, $\mathrm{H}$. et al. Laminin isoforms containing the gamma3 chain are unable to bind to integrins due to the absence of the glutamic acid residue conserved in the C-terminal regions of the gamma1 and gamma2 chains. J. Biol. Chem. 283, 28149-28157 (2008).

59. Haenggi, T. \& Fritschy, J. M. Role of dystrophin and utrophin for assembly and function of the dystrophin glycoprotein complex in non-muscle tissue. Cell. Mol. Life Sci. 63, 1614-1631 (2006). 